\title{
Plasmon bands in metallic nanostructures
}

\author{
J. E. Inglesfield, ${ }^{1}$ J. M. Pitarke, ${ }^{2,3}$ and R. Kemp ${ }^{1}$ \\ ${ }^{1}$ Department of Physics and Astronomy, Cardiff University, Cardiff, CF24 3YB, United Kingdom \\ ${ }^{2}$ Materia Kondentsatuaren Fisika Saila, Zientzi Fakultatea, Euskal Herriko Unibertsitatea, 644 Posta kutxatila, \\ E-48080 Bilbo, Basque Country \\ ${ }^{3}$ Donostia International Physics Center (DIPC) and Centro Mixto CSIC-UPV/EHU, Manuel de Lardizabal Pasealekua, \\ E-20018 Donostia, Basque Country
}

(Received 4 March 2004; published 24 June 2004)

\begin{abstract}
The photonic band structure of a three-dimensional lattice of metal spheres is calculated using an embedding technique, in the frequency range of the Mie plasmons. For a small filling factor of the spheres, the MaxwellGarnett theory gives an almost exact description of the dipole modes, and the multipole modes are fairly dispersionless. For a larger filling factor, crystal field effects modify the multipole frequencies, which show dispersion. These multipole bands are enclosed between the dipole modes. For touching spheres, there is a wide continuum of plasmon modes between zero frequency and the bulk metal plasmon frequency, which yield strong absorption of incident light. These plasmon modes are responsible for the blackness of colloidal silver.
\end{abstract}

DOI: 10.1103/PhysRevB.69.233103

PACS number(s): 42.25.Bs, 42.70.Qs, 73.22.Lp

An isolated free-electron metallic sphere shows Mie plasmons at frequencies

$$
\omega_{l}=\omega_{p} \sqrt{\frac{l}{2 l+1}},
$$

where $\omega_{p}$ is the bulk plasmon frequency, and $l$ is the quantum number identifying the multipole. This assumes the Drude dielectric function

$$
\epsilon(\omega)=1-\frac{\omega_{p}^{2}}{\omega(\omega+i / \tau)},
$$

where $\tau$ is the scattering time. In a lattice, crystal field effects shift the Mie plasmon frequencies, an effect which has been studied both experimentally and theoretically. ${ }^{1}$ These theoretical studies have generally been restricted to the dipole plasmons and their crystal field, but recently a number of methods have been developed for a full solution of Maxwell's equations in periodic structures..$^{2-6}$ There have been photonic band structure calculations of lattices of metallic rods, ${ }^{7}$ cylinders, ${ }^{8}$ and spheres. ${ }^{9-12}$ The plasmon modes and their interaction with light have been studied by Yannopapas et al. ${ }^{10}$ in the low filling fraction regime.

In this paper, we use the embedding method to solve Maxwell's equations, ${ }^{5}$ and consider the evolution of plasmon bands in a lattice of nanoscale metallic spheres as the sphere size increases from very small, practically the isolated sphere limit, to touching spheres. Our band structures and densities of states show clearly the interaction of light with such systems, and the way that the plasmons are affected by the crystal field. We find that the presence of structured metal introduces a continuum of plasmon modes, which yield strong absorption of incident light and are responsible for the unique optical properties of colloidal metals.

In the photonic embedding method, the dielectric objects-here metallic spheres-are replaced by an embedding "potential" over their surface. This is added to the wave equation for the uniform vacuum region between the spheres, and the equation is only solved explicitly in this region. The embedding potential, in reality a surface tensor operator, ensures that the surface parallel components of the $\mathbf{E}$ and $\mathbf{H}$ fields match across the boundary between the vacuum and the metal spheres. The electromagnetic field is expanded in terms of any suitable basis set (we use vector plane waves) in the region between the spheres, and because this field has no discontinuities the expansion converges very well. In the case of nonmetallic dielectric spheres, the convergence is at least an order of magnitude better than a direct plane wave expansion through the whole of space (the usual method for finding photonic band structures); $;^{13}$ in the difficult case of metallic spheres, the embedding method continues to converge well. Of course there is a price to pay, and this is the evaluation of the embedding tensor which replaces the metallic spheres. This involves finding the exact solution of Maxwell's equations inside the spheres for each multipole $l$ at frequency $\omega$, but we have derived straightforward expressions that may readily be coded.

The multipoles of the embedding tensor are cut off at a maximum value, $l_{\max }$, imposing a limit to the plasmon modes on each sphere. Without this, the density of states increases without limit as $\omega$ approaches the planar surface plasmon frequency $\omega_{p} / \sqrt{2}$ [the limit of large $l$ in Eq. (1)]. It is also necessary for convergence of the plane wave expansion. There is a physical plasmon cutoff in $l_{\max }$ due to Landau damping, though this is larger than the values we use. Controlling $l_{\max }$ also enables us to study the evolution of the plasmon bands in more detail. Unlike in scattering theory where $l_{\max }$ is similarly imposed, all higher values of $l$ are included in our embedding, but with a different boundary condition, that the surface parallel components of $\mathbf{H}$ vanish on the surface of the sphere.

The system we shall discuss here is a face-centered-cubic (fcc) lattice of metallic spheres in vacuum, the spheres being described by the Drude dielectric function of Eq. (2). The scaling property of Maxwell's equations makes it natural to use the dimensionless reduced frequency $\widetilde{\omega}=\omega a / 2 \pi c$ and reduced wave vector $\widetilde{\mathbf{k}}=\mathbf{k} a / 2 \pi$, where $a$ is the conventional 


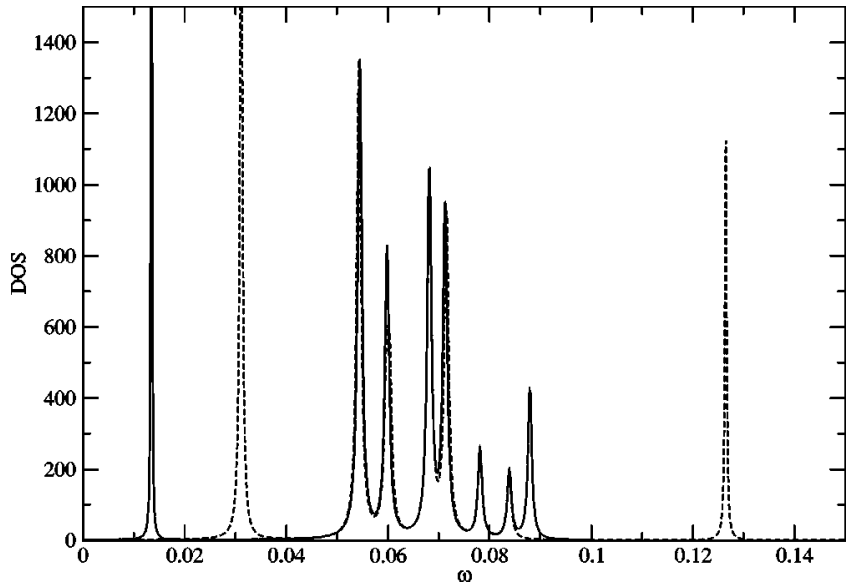

FIG. 1. $n_{\mathbf{k}}$, spectral density for fcc lattice of metal spheres, $\widetilde{r}$ $=2, \widetilde{\omega}_{p}=0.1, l_{\max }=3$, at $\widetilde{\mathbf{k}}=(0.03,0,0)$ (solid line) and $(0.1,0,0)$ (dashed line).

lattice constant. Throughout this paper we use the reduced value of $\widetilde{\omega}_{p}=0.1$; taking $\omega_{p}=15 \mathrm{eV}$ for $\mathrm{Al}$, this corresponds to a lattice constant of $a=83 \AA$. The reduced lifetime $\tilde{\tau}$ is taken to be 1000 , a value which enables us to separate spectral features. We note that quantum size effects are likely to be important in the small spheres we study here-this will be treated in later work.

In the embedding method, with a frequency-dependent embedding tensor, it is most straightforward to use a planewave expansion for the Green function between the spheres, and from this we calculate the spectral density $n_{\mathbf{k}}(\omega)$ (proportional to the energy density) integrated through the vacuum region. Figure 1 shows a typical spectral density, for a fcc lattice of metal spheres with reduced radius $\tilde{r}=2 \pi r / a$ $=2$, corresponding to an actual radius of $r=26.3 \AA$ and a filling fraction of $54 \%$, at $\widetilde{\mathbf{k}}=(0.03,0,0)$ and $(0.1,0,0)$ [the $X$ point corresponds to $\tilde{\mathbf{k}}=(1,0,0)]$. In this calculation $l_{\max }=3$, and convergence has been achieved with 18 transverse and 181 longitudinal plane waves. The number of transverse waves stays very low over the frequency range shown, but the number of longitudinal waves increases as the radius of the sphere decreases and $l_{\max }$ increases. The large number of longitudinal waves required is characteristic of plasmon systems. The almost constant features of Fig. 1 between $\widetilde{\omega}=0.055$ and 0.085 are the multipole plasmons with a nondegenerate dipole plasmon at the top of this frequency range. The dispersing peaks below and above this range are the light line coupled to a doubly degenerate dipole mode.

From the peaks in the spectral density we plot the photonic band structure. We begin by considering a fcc lattice of relatively small spheres with $\tilde{r}=0.5$, an actual radius of $6.6 \AA$ and a filling fraction of $0.8 \%$. The band structure for $l_{\max }$ $=1($ solid lines in Fig. 2) shows flat dipole modes at the Mie frequency of $\omega_{p} / \sqrt{3}$ (solid horizontal line) and the doubly degenerate light line (solid lines below and above the flat dipole modes). At the crossing point the light lines mix with two of the dipole modes, but with small spheres the interaction and the splitting are small. This calculation required 2 transverse and 1591 longitudinal plane waves for conver-

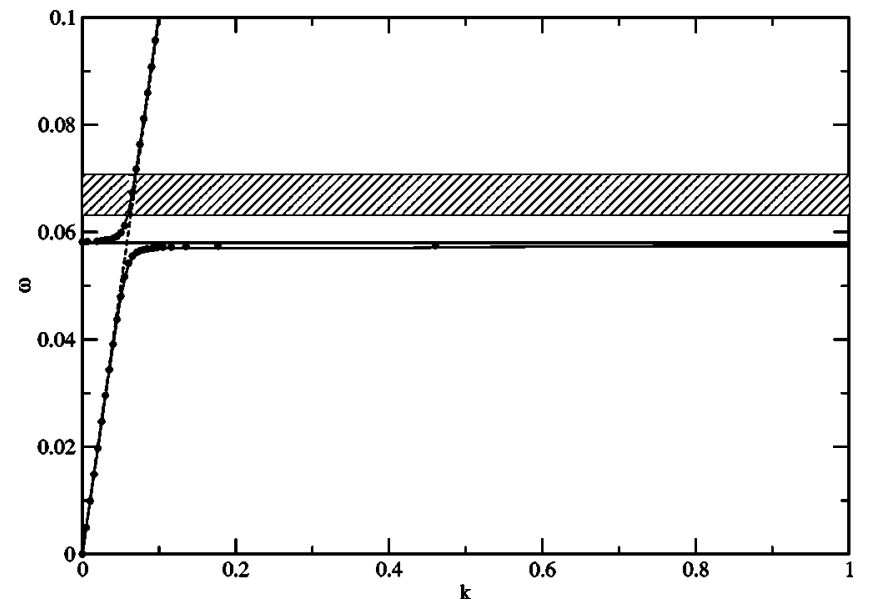

FIG. 2. Band structure for fcc lattice of metal spheres, $\widetilde{r}=0.5$, $\widetilde{\omega}_{p}=0.1$, in $\Gamma X$ direction (solid lines and shaded area). The dashed line represents the free-space dispersion relation $\widetilde{\omega}=\tilde{k}$. Filled circles are results from the Maxwell-Garnett theory.

gence. Increasing $l_{\max }$ does not change the light line and flat dipole modes, but gives the flat multipole bands at the frequencies given by Eq. (1) filling the shaded area of Fig. 2.

At low filling fractions, the interaction between the spheres can be considered within the Maxwell-Garnett approximation. ${ }^{14}$ For the light mode that we see in Fig. 2, we can define a frequency-dependent effective dielectric function

$$
\epsilon_{\text {eff }}(\omega)=\widetilde{k}^{2} / \widetilde{\omega}^{2},
$$

and the Maxwell-Garnett approximation then gives ${ }^{15}$

$$
\epsilon_{\mathrm{eff}}(\omega)=1-f \frac{1}{[1-\epsilon(\omega)]^{-1}-m}
$$

and

$$
\epsilon_{\mathrm{eff}}^{-1}(\omega)=1+f \frac{1}{[1-\epsilon(\omega)]^{-1}-n} .
$$

Here $f$ is the filling fraction, $m=(1-f) / 3$, and $n=(1+2 f) / 3$. The filled circles in Fig. 2 show the results for the dispersion calculated from Eqs. (3) and (4). There is, not surprisingly, precise agreement with our calculated results for the light dipole mode.

The concept of effective dielectric function is particularly useful for understanding optical absorption, which is dictated by the poles of $\epsilon_{\text {eff }}$. Conversely, the energy loss of fast charged particles is dictated by the poles of $\epsilon_{\text {eff }}^{-1}$. An inspection of Eqs. (4) and (5) shows that in the isolated sphere limit $(f \rightarrow 0)$ both optical absorption and energy loss occur at the Mie dipole frequency of $\omega_{p} / \sqrt{3}$. At finite filling fraction, the Maxwell-Garnett approximation predicts optical absorption at $\sqrt{m} \omega_{p}$ and energy loss at $\sqrt{n} \omega_{p}$. These are, respectively, the limit of the lower branch of the light line and the starting frequency of the upper branch.

At a sphere radius of $\widetilde{r}=1.2$, filling fraction $12 \%$, we find that the plasmon frequencies deviate from the isolated sphere results. The circles of Fig. 3 show the band structure with 


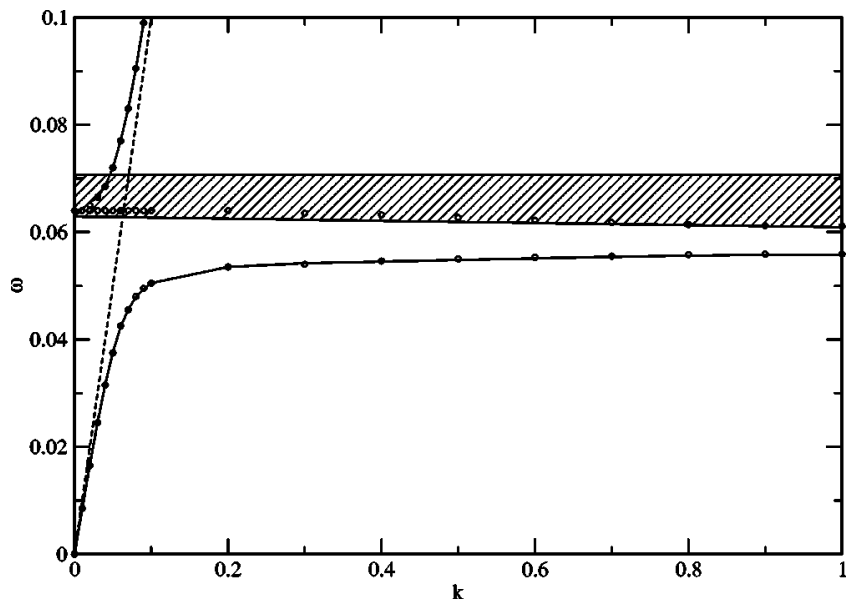

FIG. 3. Band structure for fcc lattice of metal spheres, $\widetilde{r}=1.2$, $\widetilde{\omega}_{p}=0.1, l_{\max } \rightarrow \infty$, in $\Gamma X$ direction (solid lines and shaded area). Circles are results obtained with $l_{\max }=1$. Maxwell-Garnett calculations, not represented here, nearly coincide with $l_{\max }=1$ results.

$l_{\max }=1$. We see that the interaction with the light line produces a greater splitting of the dipole modes than in Fig. 2, which is indeed accurately reproduced by Maxwell-Garnett theory. Increasing $l_{\max }$ (solid lines of Fig. 3) barely affects the lower and upper branch of the light line, only introducing virtually flat multipole modes at the Mie frequencies of Eq. (1) which fill the shaded area of Fig. 3.

A calculation of the band structure of a simple cubic lattice of Drude spheres with $\widetilde{\omega}_{p}=0.06$ and filling fraction of $12 \%$ was carried out by Pendry, ${ }^{9}$ by using a transfer-matrix scheme. We have calculated the band structure for those parameters, and have found that it has almost exactly the same form as the band structure of Fig. 3. This disagrees, however, with the band structure reported in Ref. 9, which shows flat low-energy bands absent in the band structure of Fig. 3. These probably originate from spurious modes associated with the edges and corners that are present in the transfermatrix discretization procedure.

The plasmon band structure is much more interesting at $\tilde{r}=2$, filling fraction $54 \%$, with greater interaction between all the modes. Figure 4 exhibits the band structure for $l_{\max }$ $=2$, in the $\Gamma X$ direction. We have also looked at this band structure in the $\Gamma W$ direction, and have found that along this direction all the states are nondegenerate, with eight plasmon bands (there is no $l=0$ plasmon). Figure 4 shows that the Maxwell-Garnett dispersion calculated from Eqs. (3) and (4) starts to become somewhat inaccurate, as its assumptions begin to break down. We also note that unlike in the case of smaller filling fractions, where the high multipole plasmons cut across the upper branch of the light line and lie higher than the flat dipole plasmon modes, here the dipole plasmons enclose the multipole modes.

As $l_{\max }$ increases, there are so many plasmon bands that they become difficult to track individually. However, we can see the general behavior by studying the spectral density. Figure 5 shows the spectral density at $\tilde{\mathbf{k}}=(0.5,0,0)$ for $\tilde{r}$ $=2$ and various values of $l_{\max }: 2,6,8,10$, and 12 . We see that there is very little difference in the overall structure for $l_{\text {max }} \geqslant 6$, the only difference being the large increase of the

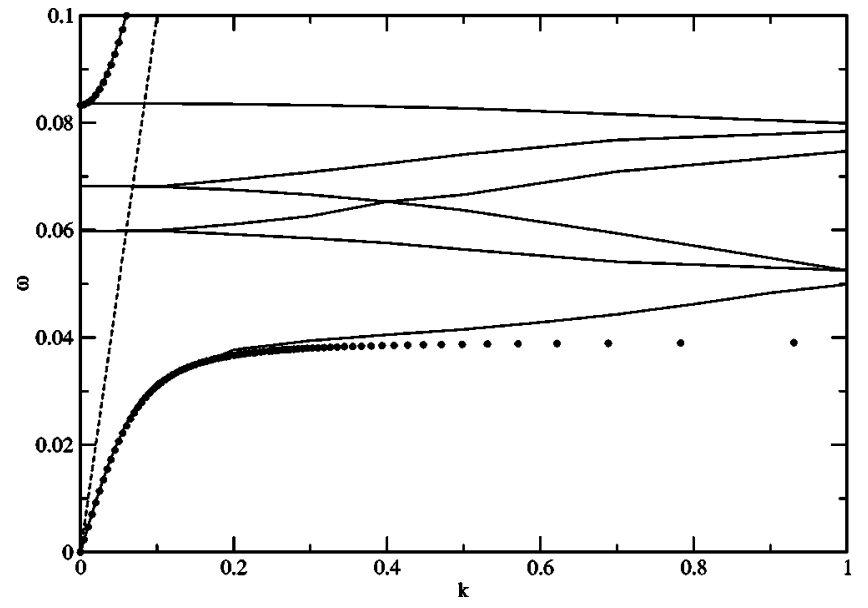

FIG. 4. Band structure for fcc lattice of metal spheres, $\tilde{r}=2.0$, $\widetilde{\omega}_{p}=0.1, l_{\max }=2$, in $\Gamma X$ direction (solid lines). Filled circles are results from the Maxwell-Garnett theory.

spectral density associated with high- $l$ multipole plasmons close to the planar surface plasmon limit at $\omega_{p} / \sqrt{2}=0.07$, in the middle of the multipole plasmon region. We can then plot the band structure in the large $l_{\max }$ limit, Fig. 6 (convergence has been achieved with $l_{\max }=8$ ), where the multipole bands lie in the shaded area enclosed by the dipole bands. The dipole bands themselves have been pushed out compared with $l_{\max }=2$ (shown in Fig. 6 by circles).

It is clear from our results for $\tilde{r}=2$, showing an increase in dipole band gap as $l_{\max }$ increases, that part of the band gap results from the interaction of the dipole modes with the multipole modes. This interaction means that the multipole modes can be excited by both photons and by fast charged particles. This contrasts with the apparent absence of interaction in Fig. 3 for $\tilde{r}=1.2$.

The behavior of the multipoles is more extreme once the spheres touch, ${ }^{16}$ corresponding to $\tilde{r}=2.2$, and a filling fraction of $74 \%$. Figure 7 shows the band structure with $l_{\max }$ $=12$. The multipole modes in the shaded area are still

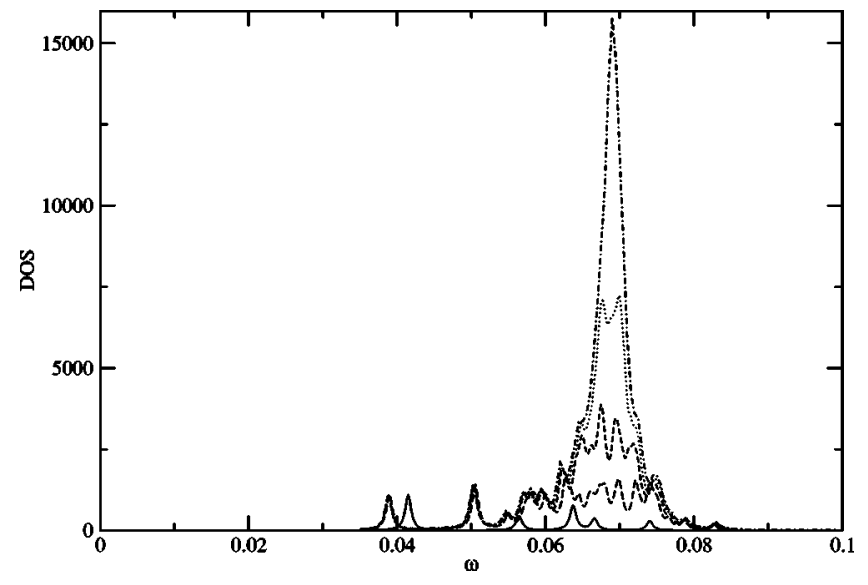

FIG. 5. $n_{\mathbf{k}}$, spectral density for fcc lattice of metal spheres, $\tilde{r}$ $=2, \widetilde{\omega}_{p}=0.1$, at $\tilde{\mathbf{k}}=(0.5,0,0)$. Solid line, $l_{\max }=2$; long-dashed line, $l_{\max }=6$; short-dashed line, $l_{\max }=8$; dotted line, $l_{\max }=10$; dasheddotted line, $l_{\max }=12$. 


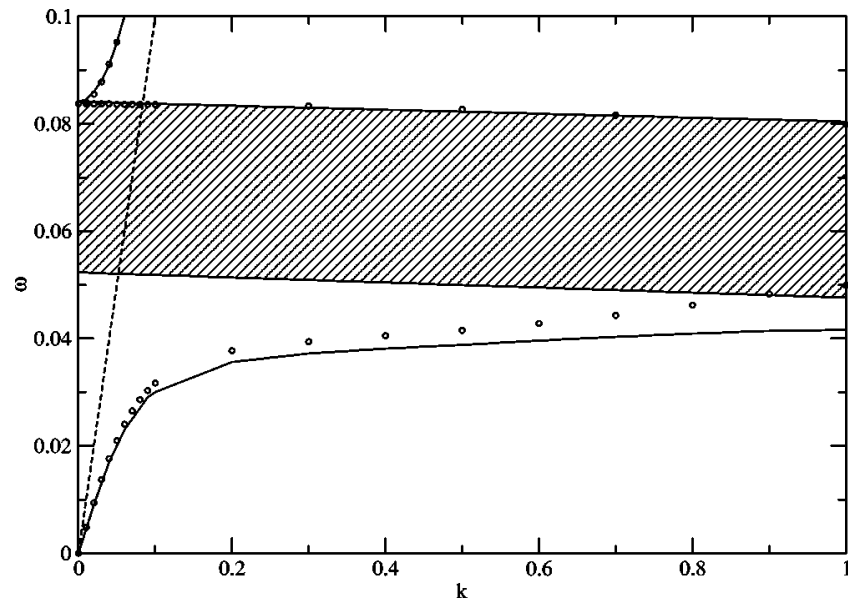

FIG. 6. Band structure for fcc lattice of metal spheres, $\widetilde{r}=2.0$, $\widetilde{\omega}_{p}=0.1, l_{\max } \rightarrow \infty$, in $\Gamma X$ direction (solid lines and shaded area). The circles show the dipole bands of Fig. 4, obtained with $l_{\max }=2$.

bounded by the dipole modes, but as $l_{\max }$ increases the multipole modes steadily broaden, pushing the lower dipole branch towards $\widetilde{\omega}=0$ and the upper branch towards $\widetilde{\omega}_{p}$. The upper branch appears to have reached its upper limit by $l_{\max }=12$, just below $\widetilde{\omega}_{p}$; the lower branch is still moving down, and it is not clear whether this reaches the zero frequency of the bulk metal. However, there is a very wide band

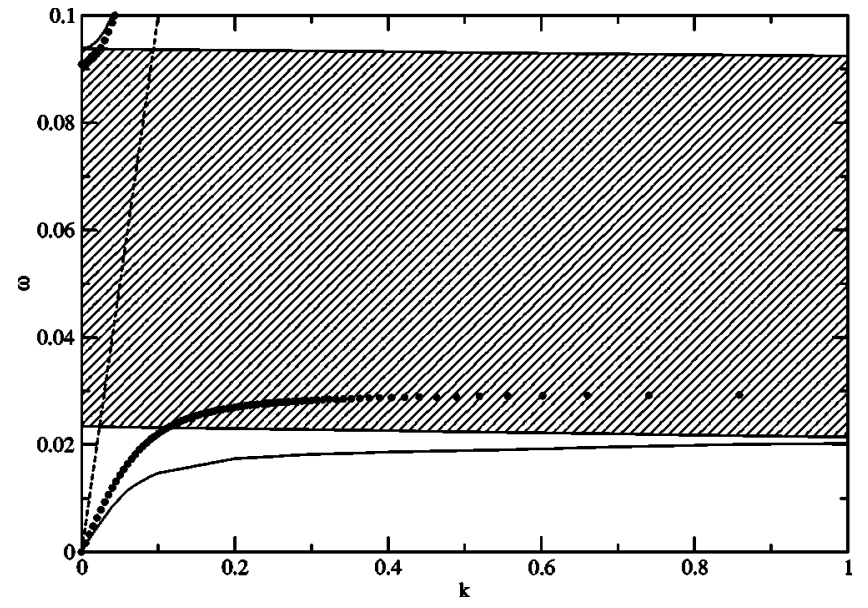

FIG. 7. Band structure for fcc lattice of touching metal spheres, $\widetilde{r}=2.2, \widetilde{\omega}_{p}=0.1, l_{\max }=12$, in $\Gamma X$ direction. Filled circles are results from the Maxwell-Garnett theory.

of multipole modes nearly spanning these limits, with corresponding optical absorption. This is the reason for the striking blackness of colloidal silver.

J.M.P. acknowledges support by the UPV/EHU, the Basque Unibertsitate eta Ikerketa Saila, the Spanish MCyT, and the UK EPSRC.
${ }^{1}$ See, e.g., D. J. Bergman and D. Stroud, Solid State Phys. 46, 147 (1992).

${ }^{2}$ E. Yablonovitch, T. J. Gmitter, R. D. Meade, A. M. Rappe, K. D. Brommer, and J. D. Joannopoulos, Phys. Rev. Lett. 67, 3380 (1991).

${ }^{3}$ K. M. Ko, C. T. Chan, and C. M. Soukoulis, Phys. Rev. Lett. 65, 3152 (1990).

${ }^{4}$ J. B. Pendry and A. MacKinnon, Phys. Rev. Lett. 69, 2772 (1992).

${ }^{5}$ J. E. Inglesfield, J. Phys. A 31, 8495 (1998).

${ }^{6}$ F. J. García de Abajo, Phys. Rev. Lett. 82, 2776 (1999).

${ }^{7}$ R. English, J. M. Pitarke, and J. B. Pendry, Surf. Sci. 454, 1090 (2000).

${ }^{8}$ H. van der Lem, J. Opt. A, Pure Appl. Opt. 2, 395 (2000).
${ }^{9}$ J. B. Pendry, J. Mod. Opt. 41, 209 (1994).

${ }^{10}$ V. Yannopapas, A. Modinos, and N. Stefanou, Phys. Rev. B 60, 5359 (1999).

${ }^{11}$ A. Moroz, Phys. Rev. Lett. 83, 5274 (1999).

${ }^{12}$ W. Y. Zhang, X. Y. Lei, Z. L. Wang, D. G. Zheng, W. Y. Tam, C. T. Chan, and P. Shing, Phys. Rev. Lett. 84, 2853 (2000).

${ }^{13}$ R. Kemp and J. E. Inglesfield, Phys. Rev. B 65, 115103 (2002).

${ }^{14}$ C. F. Bohren and D. R. Huffman, Absorption and Scattering of Light by Small Particles (Wiley, New York, 1983).

${ }^{15}$ J. M. Pitarke, F. J. García-Vidal, and J. B. Pendry, Phys. Rev. B 57, 15261 (1998).

${ }^{16}$ A. Pack, M. Hietschold, and R. Wannemacher, Opt. Commun. 194, 277 (2001). 